\title{
Vehicular Suspension and Propulsion Using Double Sided Linear Induction Machines
}

\author{
Tom Cox \\ Power Electronics Machines and Control Group \\ The University of Nottingham \\ Nottingham, UK \\ t.cox@,nottingham.ac.uk
}

\author{
Fred Eastham \\ Department of Electronic \& Electrical Engineering \\ The University of Bath \\ Bath, UK \\ jfeastham@aol.com
}

\author{
Matt Dickinson \\ Force Engineering Ltd. \\ Shepshed, UK \\ matt@force.co.uk
}

\begin{abstract}
This paper presents a new method of combined electromagnetic levitation and propulsion using a double sided pair of linear induction machines and a simple conductive sheet secondary. If the supply phase angle of one primary is modified with respect to that of the other, a controllable lift force can be developed on the conductive secondary and its load at any velocity or when stationary. Further, a resolution force is developed tending to drive the secondary into the center of the air gap, meaning that the system is inherently self-stabilizing without complex position feedback or control. This effect is studied and predicted using finite element analysis and then measured and confirmed using an experimental rig.
\end{abstract}

Keywords- Magnetic Suspension, Magnetic Levitation (MAGLEV), Linear Motors, Magnetic Bearings

\section{INTRODUCTION}

Magnetic Levitation (MAGLEV) technology aims to levitate a vehicle in order to eliminate both the contact friction and wear commonly found in wheeled vehicles. Many configurations of MAGLEV system have been theorized and developed over the years[1-3], with several systems successfully installed and operating commercially worldwide.

Maglev systems generally employ two distinct electromagnetic systems, one for levitation and one for propulsion [4]. The propulsion motor tends to be a multiphase linear machine, while the suspension system takes the form of electromagnets using attractive or repulsive forces to provide levitation.

In this paper a new method of MAGLEV is proposed, using a double sided pair of linear induction machines to provide both the levitation and thrust forces without requiring complex control. By varying the phase angle of the supply provided to one stator with respect to the other, a significant controlled levitation force can be developed on the vehicle by the double sided pair of stators, while still producing a significant thrust force.

\section{BACKGROUND}

A Linear Induction Motor (LIM) can consist of a primary winding which produces a travelling magnetic field and a simple conductive secondary which has currents induced in it by this field. These currents produce both linear thrust and a repulsive force that tends to lift the secondary away from the primary. This configuration has been used for levitation $[5,6]$ but has the drawback of a very large flux path in air leading to excessively high power requirements and limited airgaps.

If a LIM uses a conductive secondary sheet with back iron the flux path in air is considerably shortened which reduces the power requirement. However the secondary now has both an attractive force between primary field and secondary back iron and a repulsive force between primary field and secondary conductive sheet. Both of these forces vary with relative field speed and the net force on the secondary may be attractive or repulsive [7]. Where these systems have been proposed, complex control of the supply is required in order to control levitation and propulsion forces [8] and the system requires feedback from the airgap to balance the inherent instability of attractive suspension (i.e. the significant increase in attractive force as the gap length reduces).

In a double sided LIM a pair of wound primaries produces a travelling field in an air gap between them. The air gap contains a conductive sheet secondary to generate force. These machine configurations are widely employed in linear form for applications such as transport, aircraft launch [9], industrial applications and amusement rides. One of the main benefits of the double sided configuration compared to the single sided configuration is that the conductive secondary when in the center of the air gap experiences no net normal force in relation to the primaries [7]. When the conductive secondary is not centered in the air gap the double sided system develops a normal force that tends to drive the secondary back to the center of the gap, giving an undamped [10] intrinsic open loop position correction. 

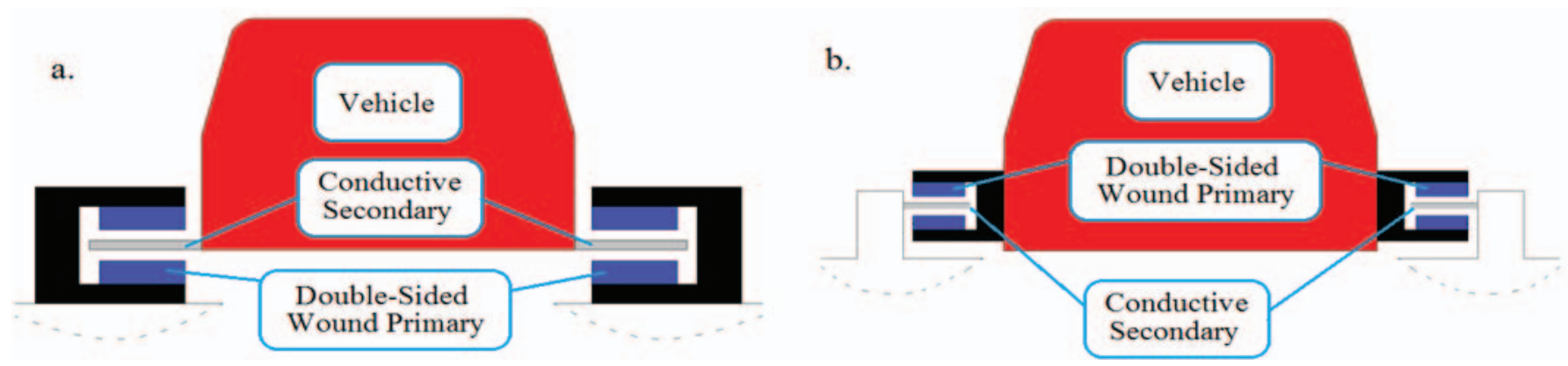

Fig. 1. Common double sided vehicle layout: a. Primaries on track, short-rotor b. Primaries on vehicle, short-stator.

Fig. 1 shows a common arrangement of LIMs as employed for transportation systems [2]. In Fig. 1a the conductive sheet secondary attached to both sides of the vehicle is driven by pairs of primaries along both sides of the track in a short-rotor configuration. This configuration minimizes the mass added to the vehicle and needs no power supply to the vehicle, but can add significant expense as wound primaries need to be placed wherever thrust is needed over the whole system. This configuration is commonly used in limited acceleration sections such as launch systems for amusement rides and aircraft.

In Fig1.b the wound primaries are mounted on the vehicle, and the track consists of a simple conductive sheet, a shortstator configuration. While this system requires a power supply to the vehicle and increases its mass, the system has only a few, fully utilized primaries and the track becomes extremely simple, inexpensive and easy to make. This type of system is ideal for transit systems, where one of the main system cost drivers is the cost and complexity of the guideway.

\section{CONCEPT}

The normal and tangential components of Maxwell stress force $F_{n}$ and $F_{t}$ on a surface are given by (1) and (2) respectively. These are calculated from the normal and tangential flux density components at that surface $B_{n}$ and $B_{t}$ and the surface area.

$$
\begin{aligned}
& F_{n}=\frac{\left(B_{n}{ }^{2}-B_{t}{ }^{2}\right)}{2 \mu_{0}} \times \text { Area } \\
& F_{t}=\frac{B_{n} B_{t}}{\mu_{0}} \times \text { Area }
\end{aligned}
$$

Where this force is calculated on a non-saturated ferrous surface it is commonly taken that the flux crossing the surface is entirely in the normal plane. Equation (3) is commonly used for simple calculation of magnetic attraction forces in a variety of electromagnetic applications by assuming $\mathrm{B}_{\mathrm{n}}=\mathrm{B}$ and $\mathrm{B}_{\mathrm{t}}=0$.

$F=\frac{B^{2}}{2 \mu_{0}} \times$ Area
On the conductive secondary of an induction machine the situation is more complex. At any field speed other than synchronized to the secondary speed, currents are induced in the secondary that contribute to the airgap flux distribution of the machine. These currents have the effect of distorting the magnetic flux across the secondary surfaces, resulting in both normal and tangential components of overall flux across the secondary surfaces.

In a double-sided pair of typical LIM primaries if the net flux distribution over the conductive secondary is considered, as shown in Fig. 2, it can be observed that the flux distribution is symmetrical on either side of the conductive secondary, with both normal and tangential flux components present at the top and bottom surfaces of the secondary.

Due to the presence of both normal and tangential flux at each secondary surface both a normal and a tangential component of Maxwell stress tensile force will also be developed, which can be calculated from (1) and (2) per surface.

As there is field symmetry along the center of the conductive secondary, the normal forces at the top and bottom surfaces of the secondary are equal and opposite and cancel each other out, while the tangential forces both produce thrust in the same direction. This effect of the double sided configuration is well known, and is commonly exploited to avoid significant attraction forces on the secondary [7].

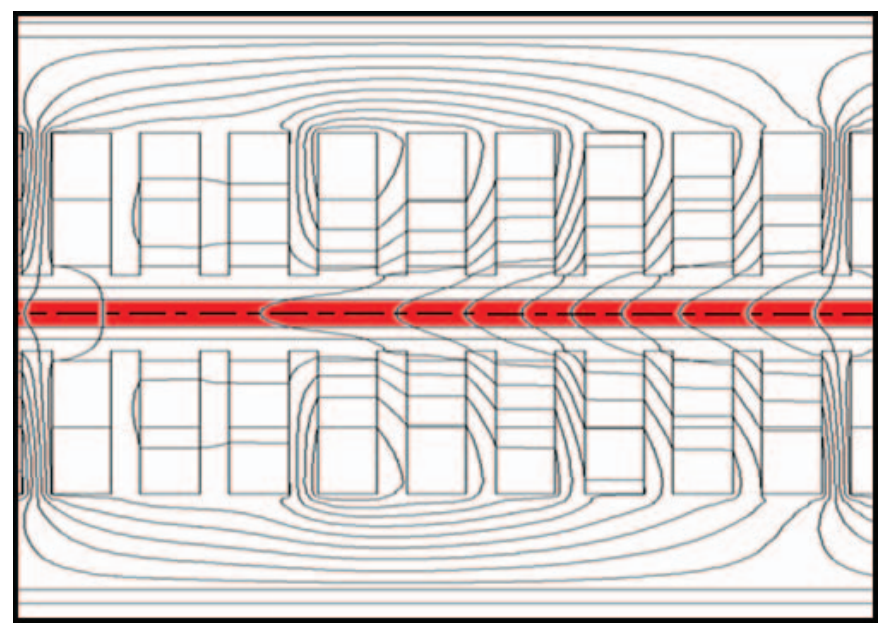

Fig. 2. Double sided machine with symmetrical field distribution. 
It can be conceived that if the air gap field normal and tangential components were non-symmetrical about the center line of the conductive secondary, the normal force across each surface would no longer be equal and opposite, giving a resultant normal force on the secondary conductor and vehicle.

Normally, a double sided pair of motors is fed from the same supply to ensure synchronized operation. If the phase angle of the three phase electrical supply of one primary in a double sided pair can be advanced or offset with respect to the other while maintaining the same supply frequency and magnitude, the net air gap flux produced by the two primaries could be made non-symmetrical about the center of the conductive secondary.

This offset angle could be introduced electrically by advancing the supply phase angle to one stator with respect to the other or physically by modifying the position of phase windings between the two stators. The two primary field excitations are otherwise synchronized together, moving in the same direction with the same peak magnitude and frequency.

\section{Electromagnetic Simulation}

The effect was initially studied using time stepped 2DFEA models of a double sided linear machine using the Infolytica MAGNET package. The model used the specification in Table I based on a machine suited to low speed transportation, but not developed specifically for levitation. The phase angle offset between the two primaries was varied over $360^{\circ}$.

\section{A. Static Thrust}

The FEA predicted static thrust and lift forces developed by the machine of Table I are shown in Fig. 3. It can be observed that at zero offset, full thrust is developed on the secondary with zero vertical force, as expected from the basic double sided configuration. At small offsets, the effect on thrust is minimal while still producing some reasonably significant vertical forces. As the field offset between the two primaries becomes larger, the negative vertical force produced peaks at $90^{\circ}$ offset then declines to zero at $180^{\circ}$ offset, while thrust reduces sinusoidally to zero at $180^{\circ}$ offset. Past this point positive vertical force occurs, peaking at $270^{\circ}$.

TABLE I

DOUBLE SidEd LiNEAR MOTOR SPECIFICATIONS

\begin{tabular}{cl}
\hline \hline Motor Parameter & Value \\
\hline winding type & Double layer \\
Slots per pole and phase & 3 \\
chording & $2 / 3$ \\
turns/coil & 28 \\
slot pitch & $19 \mathrm{~mm}$ \\
pole pitch & $171 \mathrm{~mm}$ \\
poles & 6 \\
stack width & $90 \mathrm{~mm}$ \\
total air gap (between primary faces) & $27 \mathrm{~mm}$ \\
secondary depth & $10 \mathrm{~mm}$ \\
secondary modified resistivity* & $8.24 \mathrm{e}^{-8} \Omega \mathrm{m}$ \\
secondary width mm & $300 \mathrm{~mm}$ \\
supply current per phase & $100 \mathrm{~A}_{\mathrm{rms}}$ \\
supply frequency & $50 \mathrm{~Hz}$ \\
\hline \hline
\end{tabular}

*Secondary modified resistivity includes factor of 2.009 to account for the resistance of the full secondary path including end rings in $2 \mathrm{D}$ [11]

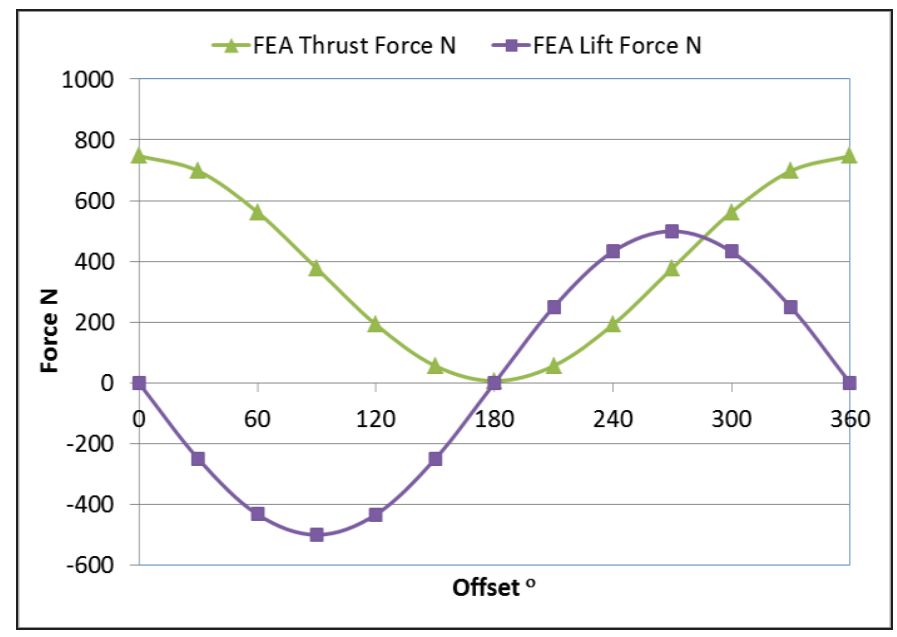

Fig. 3. Maxwell stress calculated thrust and vertical forces from FEA.

\section{B. Dynamic Thrust}

To look at how this force will behave in dynamic conditions, the 2D FEA model was rerun at $90^{\circ}$ field offset (peak lift) for all velocity conditions at fixed frequency. The thrust and lift forces from FEA are shown in Fig. 4 with a constant $100 \mathrm{~A}_{\mathrm{rms}}$ supply per phase.

It can be seen from the dynamic results that substantial forces are developed throughout the majority of the thrust speed curve. Both thrust and lift forces tail off to zero at synchronous speed, which is expected as there is no induced rotor current and so no method of force production. The machine also maintains the same thrust to lift force ratio that is fixed by the offset angle throughout the speed range.

\section{Magnetic Field Behaviour}

Fig 5 shows the variation of the magnetic field behavior in the double sided pair from $0^{\circ}$ to $180^{\circ}$ phase angle offset at various points.

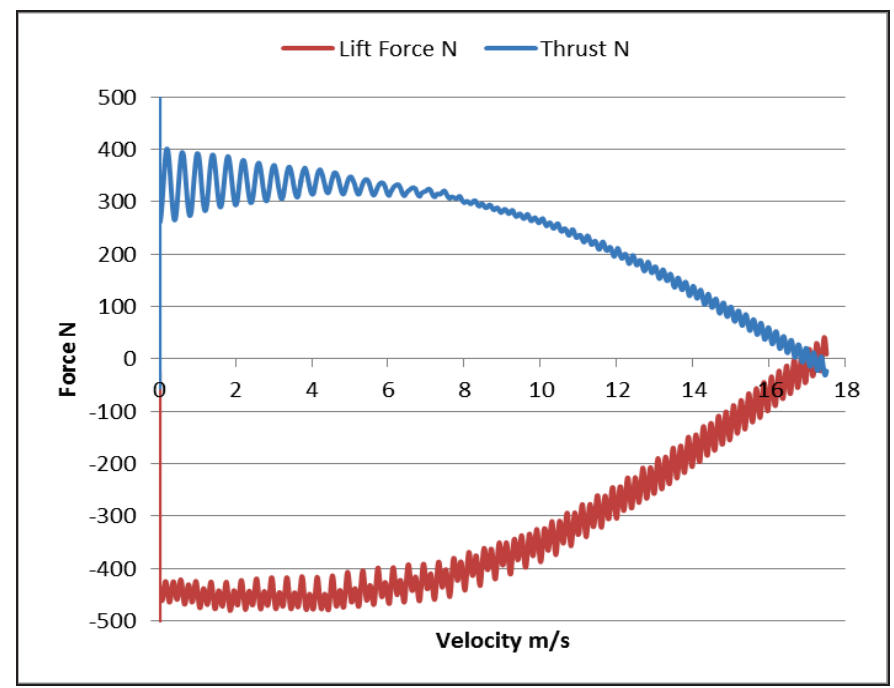

Fig. 4. Thrust and lift force variation with velocity from FEA at 90 degree phase angle offset. 


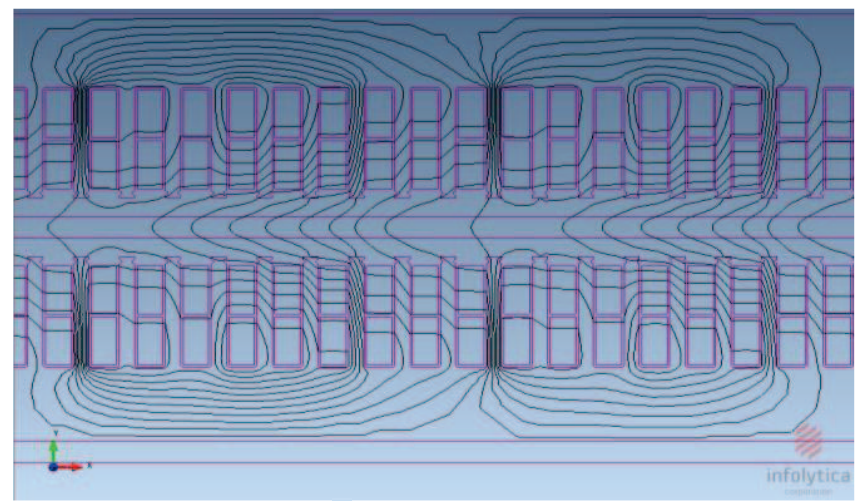

(a) $0^{\circ}$ magnetic field offset.

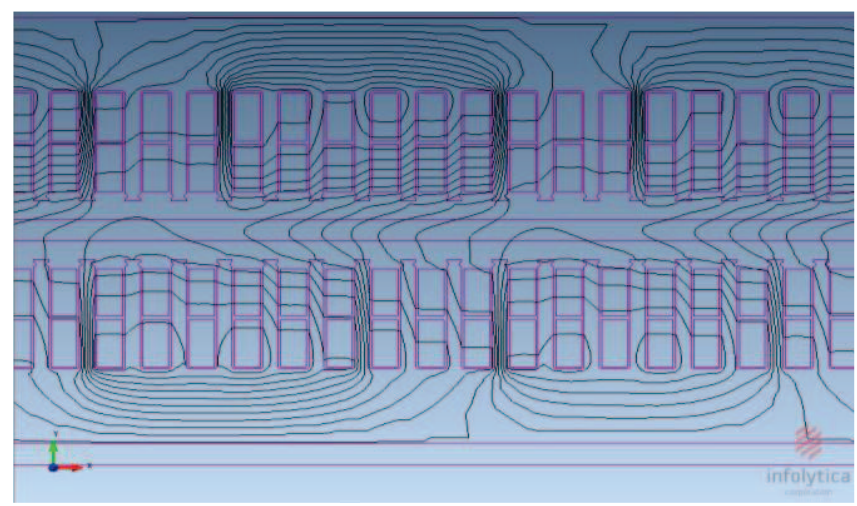

(b) $45^{\circ}$ magnetic field offset.

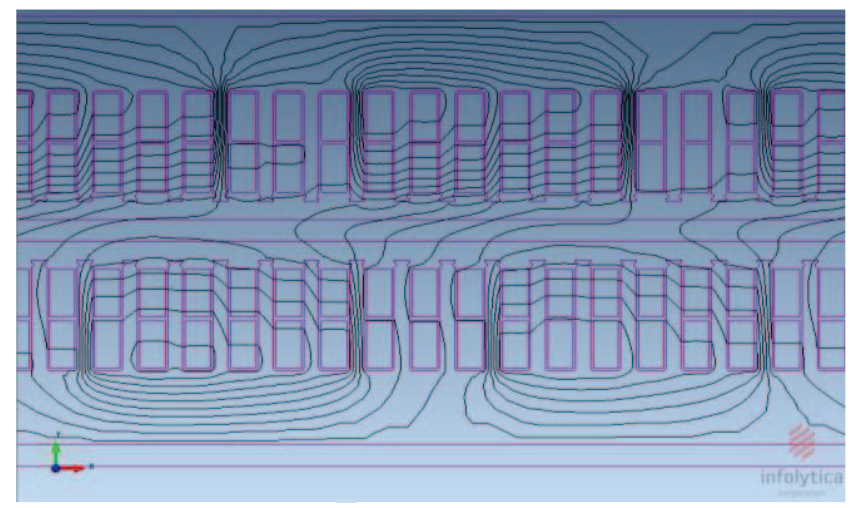

(c) $90^{\circ}$ magnetic field offset.

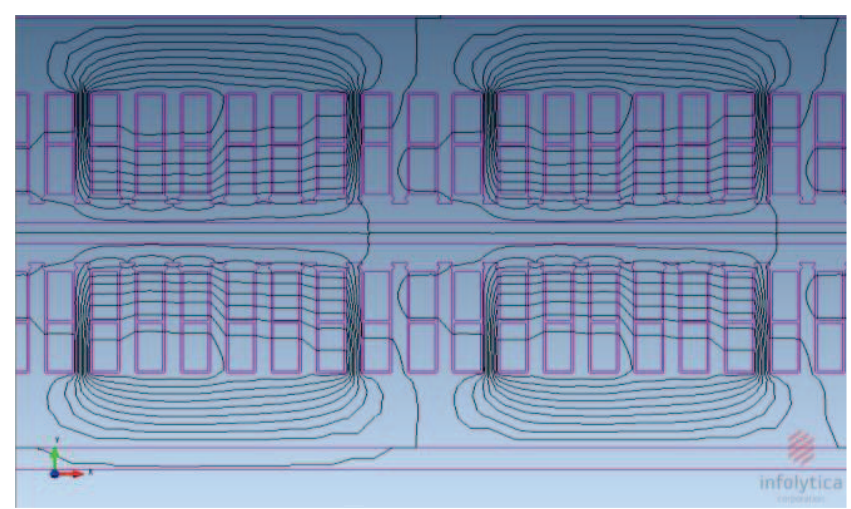

(d) $180^{\circ}$ magnetic field offset.

Fig. 5. Magnetic field contours with various phase angle offsets between double sided primaries.
At $0^{\circ}$ field offset, Fig. 5a, the field is symmetrical about the secondary as would be expected. At $45^{\circ}$ offset, Fig. 5b, some asymmetry occurs but the field magnitude is largely unchanged. At $90^{\circ}$ field offset, Fig. 5c, the field is significantly altered. Magnitude in the gap is reduced but also the field is significantly asymmetrical around the plate center point. This is the point of peak lift force from Fig. 3 .

Beyond this point, lift force from increased asymmetry in the field is a secondary factor to the overall force reduction due to a reduced air gap field. The thrust and lift forces and the air gap field magnitude all reduce to zero at $180^{\circ}$ offset, Fig. $4 \mathrm{~d}$.

\section{A. Restoring force}

The resolution force of the machine was also modelled in FEA by rerunning the static model with a secondary conductive sheet position offset of $+/-2.5$ and $+/-7.5 \mathrm{~mm}$ (of a total $8.5 \mathrm{~mm}$ clearance per side). This represents the secondary being off center in the airgap, closer to one or the other of the stators. The resolution force at stall for various offset angles is plotted in Fig. 6.

From Fig. 6 it can be seen that a significant resolution force is experienced. At $7.5 \mathrm{~mm}$ offset from center the resolution force on the secondary is $170 \mathrm{~N}$, around $1 / 3$ of the available lift force. This force will tend to drive an off center secondary towards the center of the air gap.

When used for suspension, this allows for some degree of natural load compensation. When the load on the vehicle increases, the secondary will naturally move to an off center position in the air gap where the lift force is equal to the loading. When changes in loading or position occur, the resolution force may lead to position oscillation due to low damping in the system [10]. This could be mitigated with vehicle suspension or active control of offset lift forces, or by a separate suspension system [12].

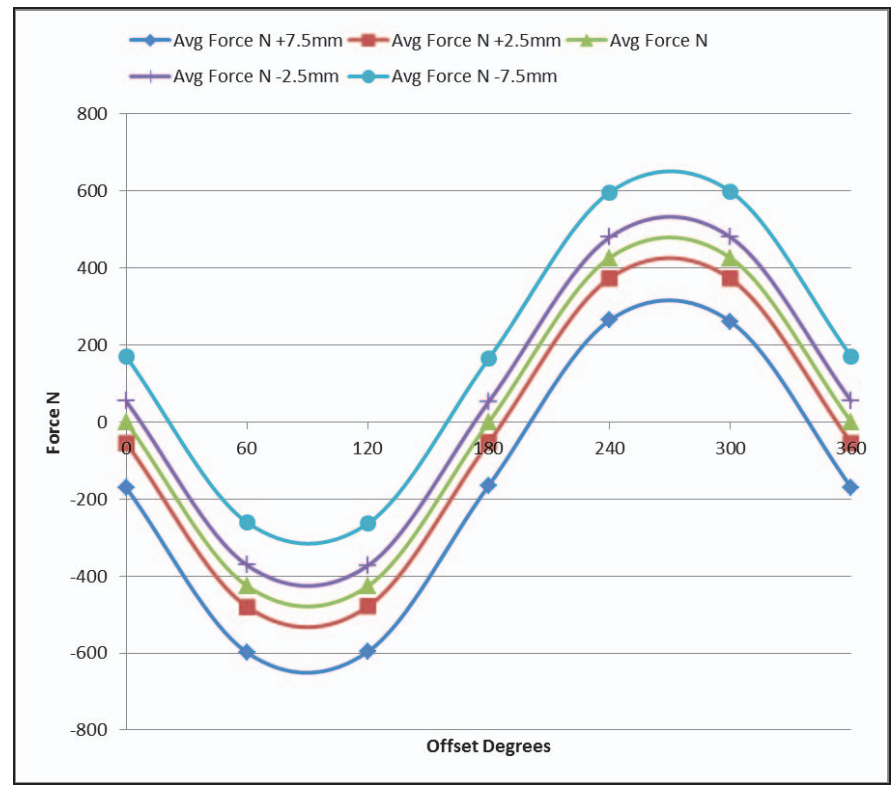

Fig. 6. Vertical force from FEA with off center conductive secondaries. 


\section{EXPERIMENTAL VALIDATION}

\section{A. Experimental Setup}

In order to verify the variation of double sided lift forces with phase angle an experimental setup was developed as outlined in Fig. 7. This used a pair of wound primaries and a conductive secondary identical to that specified in Table I, and shown in Fig. 8.

The wound primary units were mounted in a steel framework designed to withstand the large attractive force present between them.

The conductive secondary, an aluminum plate, was mechanically suspended between the two primaries in order to give a zero force condition and remove any friction component. The secondary plate was restrained by 3 linear transducers, one at the end of the secondary in the direction of thrust, and one at each end of the secondary to measure the lateral force. The lateral transducers were attached to the secondary with sliding interfaces to allow for concurrent measurement of both thrust and lateral forces.

The double sided primaries were identically wound, with each connected in a three phase delta to the same 100Arms $50 \mathrm{~Hz}$ supply. All 6 stator phase leads were accessible, and the phase angle offset was achieved by reconnecting the delta of one primary, so changing the electrical supply phase offset between the primaries in $60^{\circ}$ steps.

Measurements were repeated several times and a combination of short term activation and cooling between tests used to ensure a relatively constant secondary conductor temperature. Max test temperature was $40^{\circ} \mathrm{C}$, a $20^{\circ}$ rise from the base temperature which would correspond to a maximum change in secondary conductor resistivity of around 3\%.

In order to prove the field offset and mechanism of lift force production, a pair of pole pitch search coils was mounted onto the secondary plate, one on each face. These were used to look at differences in the field crossing the two secondary plate surfaces.

\section{B. Thrust and Lift Forces}

The thrust and lift forces developed by the motor at various offset angles were measured in $60^{\circ}$ steps over the full range of possible field offsets. The results of these force measurements are shown in Fig. 9.

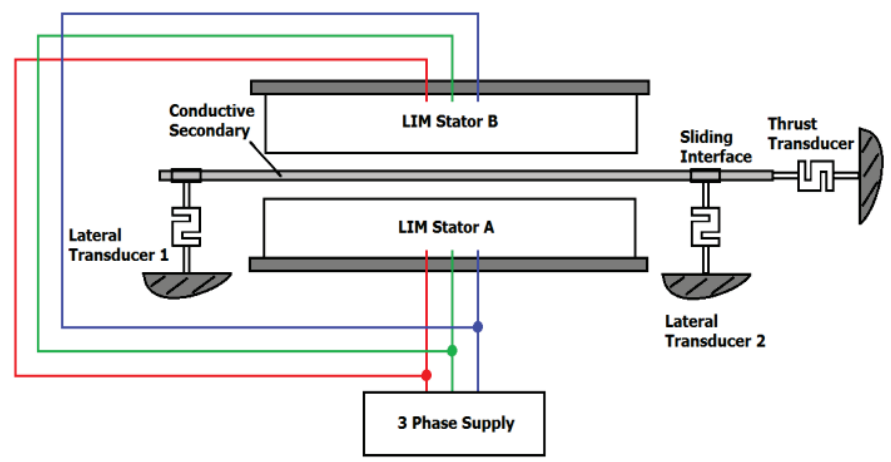

Fig. 7. System layout of test rig used to verify thrust and lift forces.

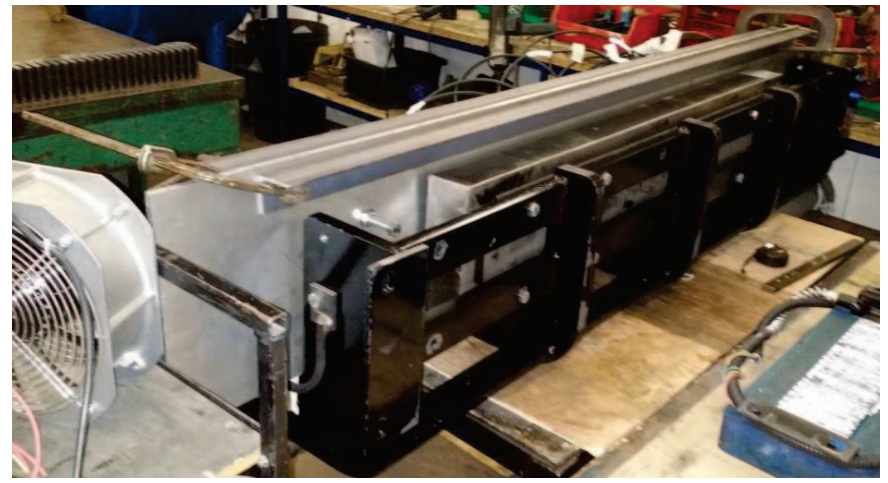

Fig. 8. Experimental setup of test rig used to verify thrust and lift forces.

It can be seen that the measured thrust and lift force at stall align with those predicted by 2DFEA, confirming the effect of offsetting to give lift force.

\section{Search coil fields}

If a flux $\Phi$ is cutting a coil at an arbitrary angle, this can be represented as components that are normal and tangential to the plane of the coil. EMF in the coil will only be generated by the normal component of the travelling field.

If search coils are placed on the top and bottom surface of a secondary conductive plate, as in Fig. 10, these coils will develop an EMF proportional to the normal component of the travelling field that is cutting the coil (and the plate surface). This allows the experimental measurement of the normal flux component across the upper and lower secondary plate surface.

In a perfectly aligned double sided pair the normal flux cutting these two coils will be equal on both sides of the secondary and so will generate an equal EMF in both coils.

As the relative phase angle of one of the primaries is changed, the Maxwell stress force on the two surfaces becomes unequal as the normal and tangential components of flux crossing the two secondary conductor surfaces vary. This difference in normal flux across the two surfaces should also be reflected in a change of the search coil measured EMF between the top and bottom coils.

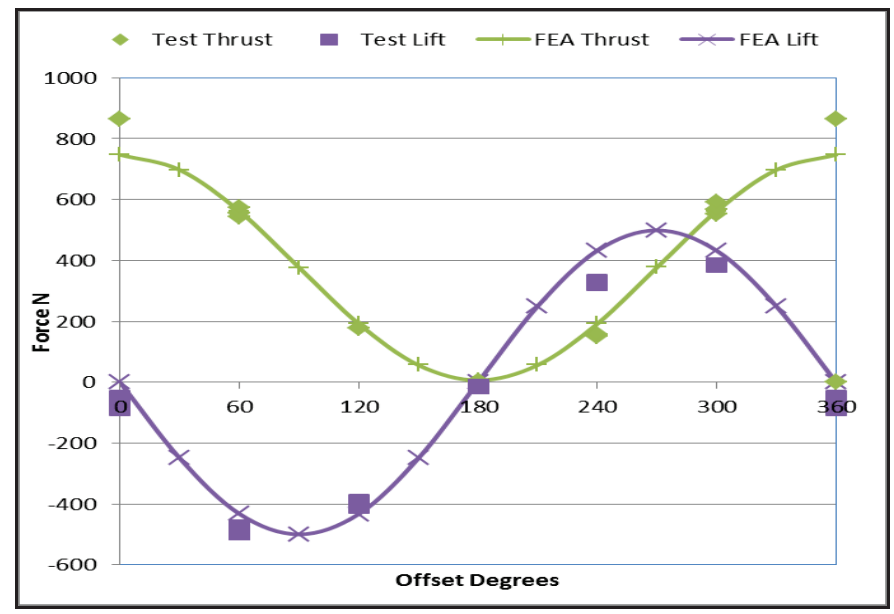

Fig. 9. Test rig thrust and lift forces compared to FEA. 


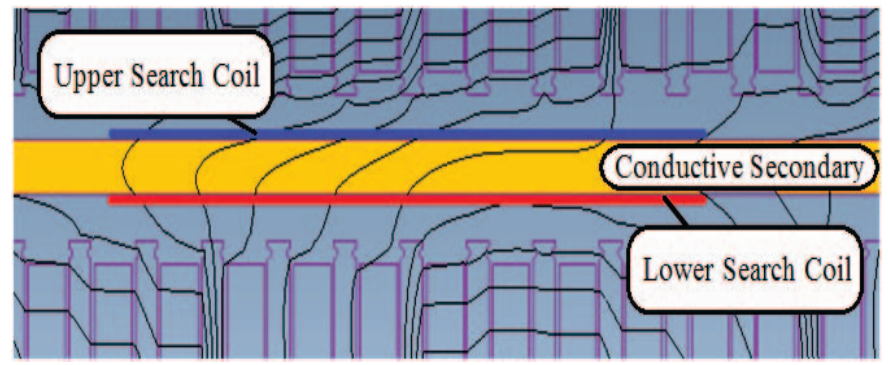

Fig. 10. Search coil configuration used to measure normal component of flux across upper and lower secondary conductive plate surface.

The normal flux is calculated in Fig. 11 from search coil measurements and is displayed vs FEA predictions. From the search coil results it can be seen that the normal flux component across the upper and lower plate surfaces varies significantly as the offset is modified.

It can further be seen that the difference in normal flux between the two surfaces is often a significant proportion of the total normal flux, at angles where lift force has been measured to be high.

Finally it is seen that there is a good correlation between FEA predicted normal flux and values experimentally measured from test rig search coil EMFs.

\section{Phase Currents}

With the overall supply current to the double sided pair kept constant at 100Arms, individual phase currents for primaries on each side of the air gap were calculated with FEA to look at any imbalance between phases or sides. In all cases the three phase currents in each primary were balanced, apart from very small variations due to linear machine end conditions. Phase current for a single phase of the upper and lower primary in the double sided pair is plotted in Fig. 12 at various phase offset angles.

It can be seen that at angles corresponding to peak values of lift force, 90 and 270 degree offsets, the currents in the top and bottom primaries are significantly imbalanced.

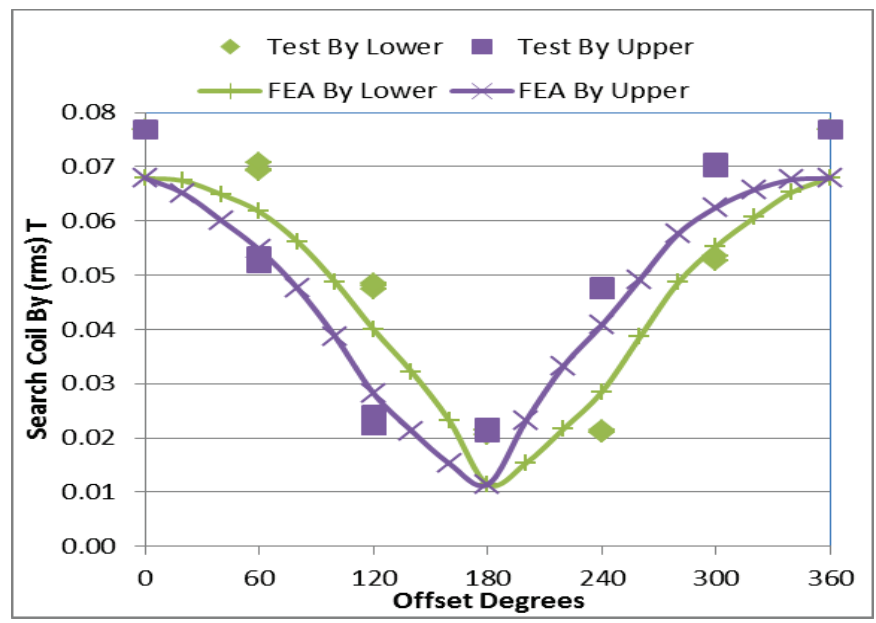

Fig. 11. Normal RMS flux at top and bottom plate surface from experimental EMF and FEA for various phase offset angles.

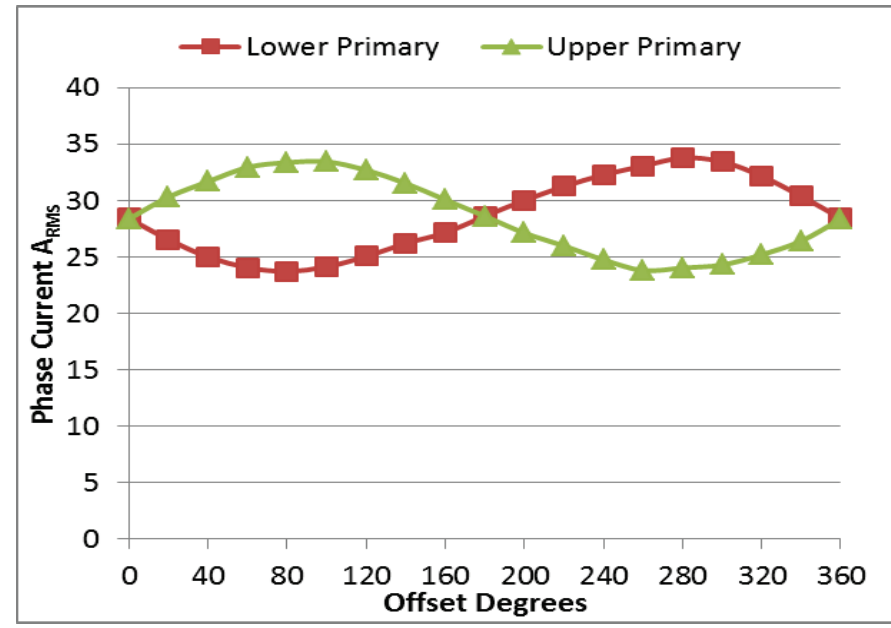

Fig. 12. Phase current imbalance between lower and upper primary windings in a double sided pair at various phase offset angles.

The overall current draw of the pair remains constant and the three phases remain in balance within each primary. However the phase current drawn by the two primaries varies by up to $+/-18 \%$ of the basic no offset current. If operated continuously in lift mode, the primaries would have to be thermally designed for the extra current in order to prevent heating problems.

\section{CONCLUSIONS}

It has been shown that offset fields in double sided machines can be used to produce significant controlled levitation forces through a set imbalance in the airgap flux normal and tangential components at the top and bottom surfaces of a conductive sheet secondary.

This force can be a significant proportion of the full thrust force. This effect can be built in with a pre-selected electrical offset angle between the two primary windings. Alternatively, power converters can be used to modify the phase angles of one primary winding supply with respect to the other in order to produce a field offset between the two primaries of any required angle, allowing dynamic control of the produced lift force.

The benefits of this effect for magnetic levitation are significant. By controlling the lift force experienced on a vehicle, frictional losses and associated wear can be significantly reduced, loads can be balanced and the vehicle may even be levitated using the vertical force provided by the primary arrangement.

A potentially significant use of this topology would be in combination with an electrodynamic suspension system using permanent magnets. Electrodynamic suspension only works effectively at speed, and electrodynamic suspension systems generally require wheels or other means of support for low speed. A system could be envisaged with a simple conductive sheet secondary track and a vehicle including both permanent magnets and a double-sided linear primary. The double sided machine can be used for propulsion of the vehicle at all speeds. When the electrodynamic lift from the permanent magnets is insufficient to lift the vehicle (zero to low speed), offset field 
levitation can be used to levitate the vehicle. As the vehicle accelerates a greater proportion of lift will come from the permanent magnet system and the field offset can be reduced to compensate. The double sided machine will exert a force tending to keep the conductive secondary centered in the air gap, and the field angle can also be adjusted to provide a lift force to compensate for variations in loading or to control oscillations. Using the lift mode for run up/run down and short term operation would also mean that the thermal imbalance due to uneven current distribution between the double sided primaries could likely be neglected.

[1] S. Yamamura, "Magnetic levitation technology of tracked vehicles present status and prospects," IEEE Transactions on Magnetics, vol. 12, pp. 874-878, 1976.

[2] L. Hyung-Woo, K. Ki-Chan, and L. Ju, "Review of maglev train technologies," IEEE Transactions on Magnetics, vol. 42, pp. 19171925, 2006.

[3] R. Hellinger and P. Mnich, "Linear Motor-Powered Transportation: History, Present Status, and Future Outlook," Proceedings of the IEEE, vol. 97, pp. 1892-1900, 2009.

[4] W. W. Dickhart, "Status Of Transrapid Maglev," in Electro International, 1991, 1991, pp. 613-617.

[5] J. F. Eastham and D. Rodger, "The performance of induction levitators," Magnetics, IEEE Transactions on, vol. 20, pp. 16841686, 1984.

[6] J. Eastham and S. Williamson, "Experiments on the lateral stabilisation and levitation of linear induction motors," IEEE Transactions on Magnetics, vol. 10, pp. 470-473, 1974.

[7] B.-t. Ooi and D. White, "Traction and Normal Forces in the Linear Induction Motor," IEEE Transactions on Power Apparatus and Systems, vol. PAS-89, pp. 638-645, 1970.

[8] K. Tsuruya, T. Morizane, N. Kimura, and H. Omori, "Simultaneous thrust and attractive force control of linear induction motor driven by power source with frequency component synchronous with motor speed," in Power Electronics and Applications (EPE'15 ECCE-Europe), 2015 17th European Conference on, 2015, pp. 110.

[9] J. Proverbs, T. Cox, and J. F. Eastham, "Parallel and flux forced windings in discontinuous machines," in Electric Machines \& Drives Conference (IEMDC), 2011 IEEE International, 2011, pp. 527-532.

[10] D. Rodger and J. F. Eastham, "Dynamic behavior of linear induction machines in the heave mode," Vehicular Technology, IEEE Transactions on, vol. 31, pp. 100-106, 1982.

[11] R. L. Russell and K. H. Norsworthy, "Eddy currents and wall losses in screened-rotor induction motors," Proceedings of the IEE Part A: Power Engineering, vol. 105, p. 163, 1958.

[12] R. Goodall, "The theory of electromagnetic levitation," Physics in Technology, vol. 16, p. 207, 1985. 